\title{
The Impact of Postdischarge Telephonic Follow-Up on Hospital Readmissions
}

\author{
Patricia L. Harrison, MPH, Pamela A. Hara, BSN, MBA, James E. Pope, MD, \\ Michelle C. Young, BS, and Elizabeth Y. Rula, PhD
}

\begin{abstract}
Recurrent hospitalizations are responsible for considerable health care spending, although prior studies have shown that a substantial proportion of readmissions are preventable through effective discharge planning and patient follow-up after the initial hospital visit. This retrospective cohort study was undertaken to determine whether telephonic outreach to ensure patient understanding of and adherence to discharge orders following a hospitalization is effective at reducing hospital readmissions within 30 days after discharge. Claims data were analyzed from 30,272 members of a commercial health plan who were discharged from a hospital in 2008 to determine the impact of telephonic intervention on the reduction of 30-day readmissions. Members who received a telephone call within 14 days of discharge and were not readmitted prior to that call comprised the intervention group; all other members formed the comparison group. Multiple logistic regression was used to determine the impact of the intervention on 30-day readmissions, after adjusting for covariates. Results demonstrated that older age, male sex, and increased initial hospitalization length of stay were associated with an increased likelihood of readmission $(P<0.001)$. Receipt of a discharge call was associated with reduced rates of readmission; intervention group members were $23.1 \%$ less likely than the comparison group to be readmitted within 30 days of hospital discharge $(P=0.043)$. These findings indicate that timely discharge follow-up by telephone to supplement standard care is effective at reducing near-term hospital readmissions and, thus, provides a means of reducing costs for health plans and their members. (Population Health Management 2011;14:27-32)
\end{abstract}

\section{Introduction}

$\mathbf{R}$ EADMISSION TO THE HOSPITAL within a short period following hospital discharge is a common and costly phenomenon, particularly within the chronically ill population. ${ }^{1,2}$ Most of these readmissions are the result of chronic disease progression in combination with inadequate postdischarge care, which is a primary factor associated with preventable readmissions. ${ }^{3}$ New or improved routes for following up with patients after they are discharged are clearly needed, based on the recent finding that $22 \%$ of patients admitted to general medicine services either die, are readmitted, or visit an emergency room within 30 days of discharge. ${ }^{4}$ Similarly, a study of nearly 12 million Medicare beneficiaries who were discharged from a hospital found that approximately one fifth were readmitted within 30 days, and that only $10 \%$ of these readmissions were planned. ${ }^{5}$

The high readmission rates experienced in the American health care system are generally attributed to inadequate communication with the patient and among the patient's doctors at the time of discharge, and a failure of physicians to follow up after a discharge, ${ }^{6,7}$ as evidenced by the fact that over half of patients who were rehospitalized within 30 days did not visit a physician's office between the 2 admissions. ${ }^{5}$ Although substandard quality of care during an initial hospitalization is often raised as a reason for repeated admissions, research is inconclusive about the relative risk contributed by this factor versus inadequate follow-up. ${ }^{3}$

Although there are no precise criteria to determine which readmissions are categorized as preventable ${ }^{8}$ the promising results of primary care initiatives to reduce readmissions are indicative of the fact that (1) many readmissions can, in fact, be avoided, and (2) focused interventions with this aim can be successful. For example, a review of results from randomized trials found that patient assessments, education, and improved postdischarge care could reduce readmission rates by $12 \%-75 \% .^{3}$ However, hospitals and clinics are challenged to meet growing demands with limited

Healthways Center for Health Research, Franklin, Tennessee. 
resources, ${ }^{9,10}$ indicating a need for ancillary providers of such services.

The financial consequences of preventable readmissions are staggering. In 2004, Medicare expenditures for unplanned readmissions were $\$ 17.4$ billion. ${ }^{5}$ On an individual basis, the 6-month costs of preventable readmissions averaged $\$ 10,870$ for each readmitted patient across 4 states in 1999. ${ }^{11}$ The Medicare Payment Advisory Commission has alerted Congress of the magnitude of this problem and made recommendations for policy that would encourage hospitals to adopt measures to reduce readmissions, recognizing that other providers can be instrumental in achieving the desired outcome. $^{12}$

With the aim of reducing hospital readmissions, we delivered a specialized telephonic intervention to health plan members who were discharged from the hospital. The discharge calls, delivered by trained nurses, provided education and support for appropriate follow-up steps. In this study, we first examined the variables associated with hospital readmission to elucidate patient characteristics that indicate the greatest need of postdischarge support. We then evaluated whether patients who received a call within 14 days of discharge had reduced 30-day hospital readmission rates in comparison to discharged patients who did not receive the intervention. The results of this study indicate that telephonic discharge follow-up offers an effective method to reduce the burden associated with preventable readmissions.

\section{Methods}

\section{Eligibility criteria}

Participants in this study included all 30,272 members from a large commercial health plan with Medicare Advantage who were enrolled in a chronic disease management program and who had a hospital admission for any reason during calendar year 2008. Eligibility for a disease management program required a diagnosis of asthma, coronary artery disease, chronic kidney disease, chronic obstructive pulmonary disease, depression, diabetes, end-stage renal disease, heart failure, or one of 11 other chronic conditions that frequently present as comorbid conditions and are often associated with increased utilization. Program enrollment was automatic for eligible members unless the individual elected to opt out of the program. Member inclusion in this study was irrespective of the type of disease management program in which they were enrolled, the primary diagnosis during initial hospitalization, or the location to which members were discharged. All analyses were retrospective in nature; thus, the treatment of participants was not altered in any way for the purposes of this study.

\section{Intervention}

The Hospital Discharge Campaign (HDC) was a focused intervention aimed at reducing preventable hospital readmissions and delivered within chronic disease management programs. Program members were entered into the HDC upon the health plan notifying the program provider of a discharge following an inpatient hospital stay. Members were then eligible to receive a telephone call from a speciallytrained registered nurse to verify that the member, or the member's caregiver, received discharge instructions, did not receive duplicate or contraindicated prescriptions for medications, and understood the proper steps to take, such as follow-up physician visits, to avoid additional acute events or exacerbations.

\section{Assessment of outcomes}

The first occurrence of a hospital admission for each member within the 2008 calendar year was considered the initial hospital admission for that member. A subsequent hospital admission for any reason that occurred within 30 days of the initial hospital discharge was considered a 30-day readmission. Hospital admissions and/or readmissions that occurred more than 30 days after the initial hospital discharge were not included in this study.

\section{Classification of members}

Members who received discharge calls within 14 days of their initial hospital discharge were classified as having received the intervention. The 14-day requirement was imposed because the first 2-3 weeks after discharge are considered the critical window for prevention of readmissions. ${ }^{13}$ The exceptions were members who were readmitted prior to receiving a discharge call; these members, who comprised only $0.5 \%$ of the total study population, were assigned to the comparison group because a discharge call must precede a readmission to have the opportunity to prevent that readmission. Members who did not receive a discharge call within 30 days of initial hospital discharge and those members who received discharge calls between days 15 and 30 following their initial hospital discharge were also assigned to the comparison group. The intervention group $(\mathrm{N}=6773)$ and the comparison group $(\mathrm{N}=23,499)$ were further classified by readmission status in the 30-days after discharge. The method of classification and the number of participants within each subgroup are illustrated in Figure 1.

\section{Statistical methods}

Fisher's exact test was used to assess the association between sex and whether or not members were readmitted to the hospital within 30 days of discharge. The Cochran-Armitage test was used to test for trends in the proportions of members with 30-day readmissions across levels of all ordinal covariates. Multiple logistic regression was used to determine the impact of hospital discharge calls on preventing 30-day readmissions after controlling for covariates including sex, age, length of stay (LOS) during the initial hospitalization, and a variable coined the "readmission window," which represented the varying window of opportunity members had to be readmitted to the hospital based on whether or not, and when, they received a discharge call (Fig. 2). LOS during the initial hospitalization was used as a proxy for disease severity because clinical risk is associated with the number of days spent in the hospital. ${ }^{14}$ The readmission window was 30 days for all members of the comparison group; however, because the length of the readmission window for members in the intervention group varied between 16 and 29 days based on when discharge calls were received, it was necessary to adjust for this disparity to ensure equal comparison of the two groups. All 
FIG. 1. Classification of study population into intervention and comparison groups. Shading indicates the final classification of a population subgroup; all percentages are in relation to the total population.

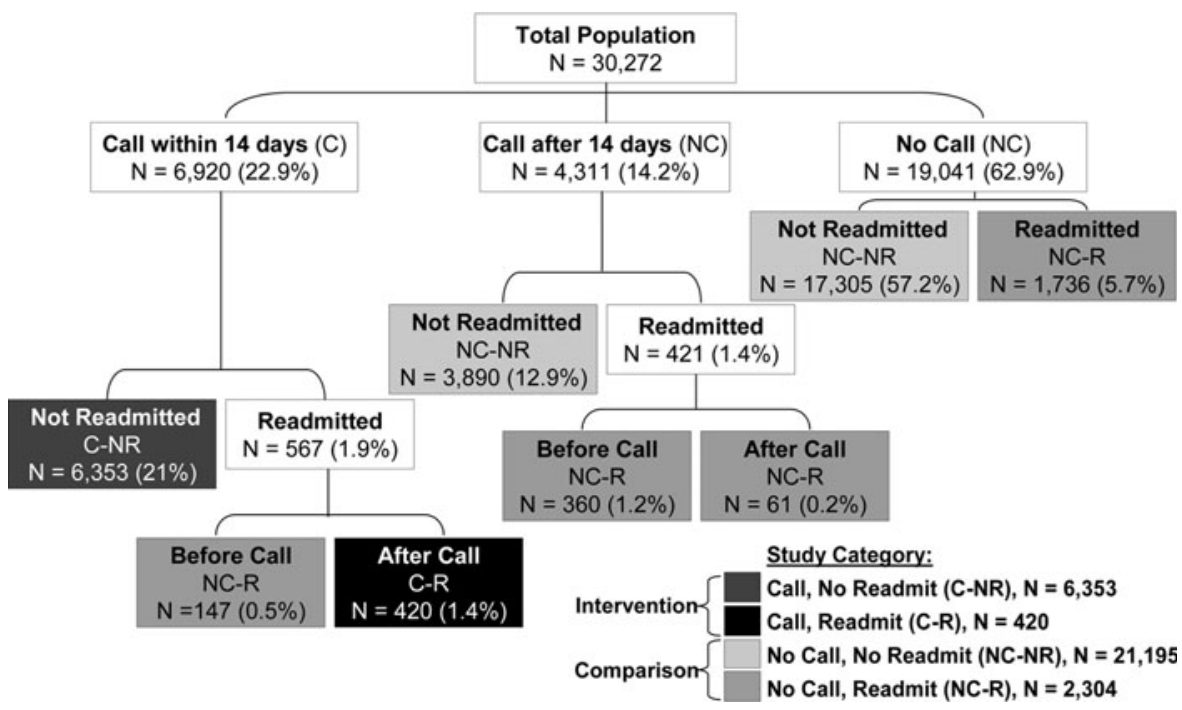

statistical analyses were conducted using SAS Release 9.1.3 (SAS Institute Inc., Cary, NC).

\section{Results}

An analysis of the specific impact of covariates across the entire eligible population $(\mathrm{N}=30,272)$ on the likelihood of readmission found that 30 -day readmissions were positively associated with older age at the time of initial hospitalization, male sex, and longer initial hospitalization LOS $(P<0.001$ for all covariates; Table 1$)$. Among all members who were readmitted during the 30 -day window $(\mathrm{N}=2724)$, the median time to readmission was 11 days. The readmission rate was highest on days 2 and 3 after discharge and declined gradually from day 4 onward. Overall, nearly a third of readmissions in the population occurred within 7 days, and over half occurred within 14 days of discharge (Fig. 3A).
Evaluation of calls by day in the intervention group $(\mathrm{N}=6773)$ found that discharge calls were received as early as 1 day following the initial hospital discharge, while the median time to discharge call was 7 days. The distribution of intervention group participants by day of call is shown in Figure 3B.

Results of a multiple logistic regression analysis with adjustment for age, sex, initial hospitalization LOS, and readmission window indicated that members who do not receive a call within 14 days after discharge are 1.3 times more likely to be readmitted to the hospital within 30 days of discharge than those who do receive calls $(P=0.043$, Table 2$)$. This odds ratio is consistent with the intervention group being $23.1 \%$ less likely to be readmitted than the comparison group. The interaction between age and initial hospitalization LOS was also examined in conjunction with the other covariates in the model, but was found to have no effect on the relationship between discharge calls and 30-day
FIG. 2. Illustration of readmission window variable.
Comparison Group $\rightarrow$ Readmission Window $=30$ Days

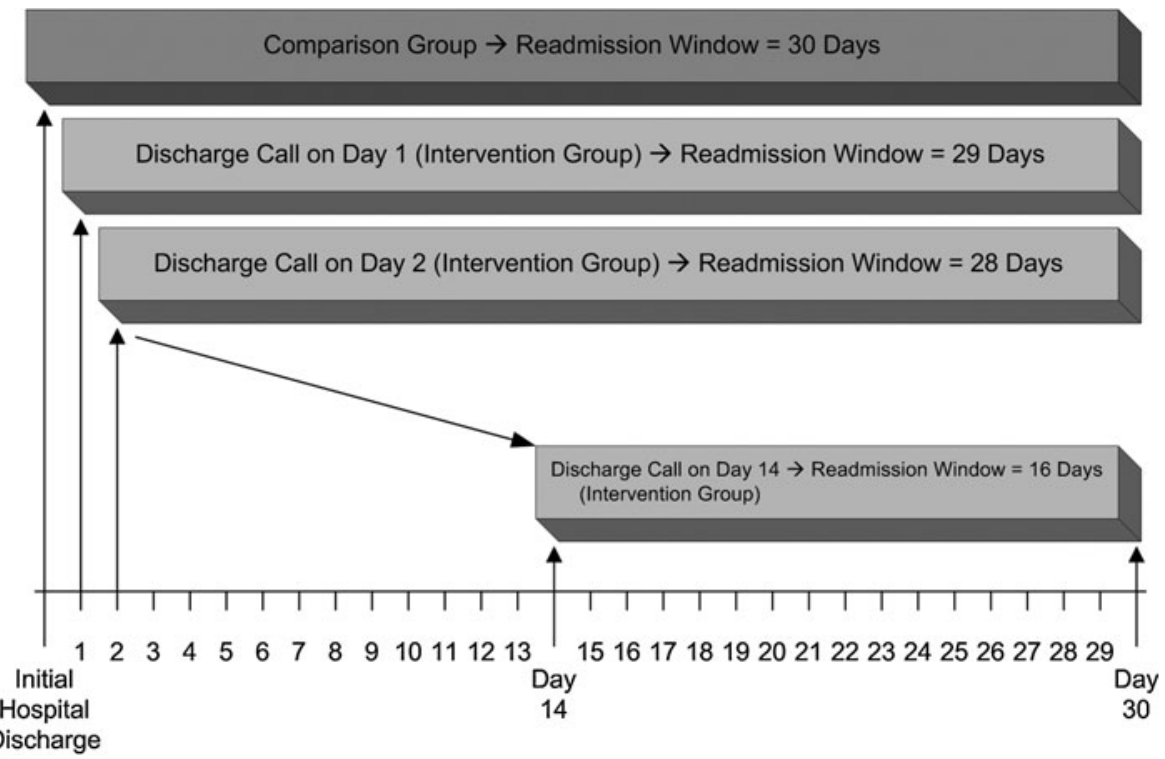


Table 1. Association between Member and Hospitalization Characteristics AND 30-DAY READMISSIONS

\begin{tabular}{|c|c|c|c|c|}
\hline Variable & Category & $\begin{array}{c}\text { Total } \\
\mathrm{N}\end{array}$ & $\begin{array}{c}\text { Percent } \\
\text { Readmitted } \\
\text { Within } 30 \text { Days }\end{array}$ & $\mathrm{P}$ value \\
\hline \multirow{5}{*}{$\begin{array}{l}\text { Age at } \\
\text { hospitalization } \\
\text { (in years) }\end{array}$} & $<18$ & 831 & $5.4 \%$ & $<0.001^{*}$ \\
\hline & $18-34$ & 3059 & $6.6 \%$ & \\
\hline & $35-49$ & 6295 & $7.9 \%$ & \\
\hline & $50-64$ & 12,622 & $9.0 \%$ & \\
\hline & $65+$ & 7465 & $11.3 \%$ & \\
\hline \multirow[t]{2}{*}{ Sex } & Female & 16,236 & $8.0 \%$ & $<0.001^{* *}$ \\
\hline & Male & 14,036 & $10.1 \%$ & \\
\hline \multirow{5}{*}{$\begin{array}{l}\text { Initial } \\
\text { hospitalization } \\
\text { length of stay } \\
\text { (in days) }\end{array}$} & 1 & 6967 & $6.7 \%$ & $<0.001^{*}$ \\
\hline & 2 & 6911 & $6.9 \%$ & \\
\hline & 3 & 5954 & $8.0 \%$ & \\
\hline & 4 & 3868 & $8.5 \%$ & \\
\hline & $5+$ & 6572 & $14.8 \%$ & \\
\hline
\end{tabular}

* $P$ values determined using the Cochran-Armitage test for trend. ** $P$ value determined using Fisher's exact test.

readmissions (data not shown). Thus, results from the more parsimonious model containing only main effects are reported here.

\section{Discussion}

The unsustainable growth in health care expenditures attributed to chronic disease highlights the need for programs that can reduce unnecessary spending. ${ }^{15}$ Programs that can achieve this goal while also improving health and quality of life are clearly of greatest consequence. Hospital readmissions are an obvious focus for such programs due to the great expense they generate, ${ }^{8}$ although a significant proportion of these could be prevented through improved care and follow-up postdischarge. Public recognition of this fact by Congress highlighted the need for additional research on the effectiveness of initiatives aimed at reducing readmission rates. $6,8,12$
Although a link has been established between substandard care during the index hospitalization and the likelihood of readmission, this is only 1 aspect of the problem. ${ }^{3}$ Research indicates that there is a direct correlation between a lack of effective discharge planning-including education, communication with the patient and family, and support after discharge-and a greater likelihood of readmission. ${ }^{16}$ This problem is further exacerbated by increasing prevalence and severity of chronic disease, ${ }^{17}$ which is the greatest indicator of readmission. ${ }^{13}$ For these two reasons, a hospital discharge call campaign was developed specifically for individuals with chronic conditions.

Among all members of the health plan in this study who were hospitalized in 2008, the data clearly show that male sex, older age, and greater initial LOS are associated with an increased likelihood of readmission. The fact that increased initial LOS is a highly significant predictor is interesting because there is evidence to suggest that premature discharge is often responsible for readmissions. ${ }^{18}$ However, a longer stay is also indicative of greater severity of the event that precipitated the initial hospitalization, ${ }^{19}$ and logically, complex cases are more likely to trigger readmissions in the absence of proper follow-up care. This finding demonstrates that these factors may be taken into consideration when identifying individuals in greatest need of postdischarge follow-up.

The hospital discharge intervention described here was developed with the combined purpose of improving quality of care, promoting health, and reducing costs by helping individuals with chronic disease adhere to their physicians' care plans, obtain appropriate standard care, and engage in self-management of their condition to reduce the likelihood of readmissions. Our analysis of the intervention demonstrates that it was successful in this objective, as evidenced by the reduction in readmissions among intervention group members. The reduction in readmissions was significant even after statistical adjustment for factors that were identified as increasing the likelihood of readmission.

To estimate the financial impact of this program, the average cost of a preventable readmission was applied to the readmissions that were avoided by the intervention group. This analysis suggests that discharge calls to members included in this study resulted in savings of approximately \$1.4 million (Table 3). Furthermore, this value is likely an underestimate for calendar year 2008 because any
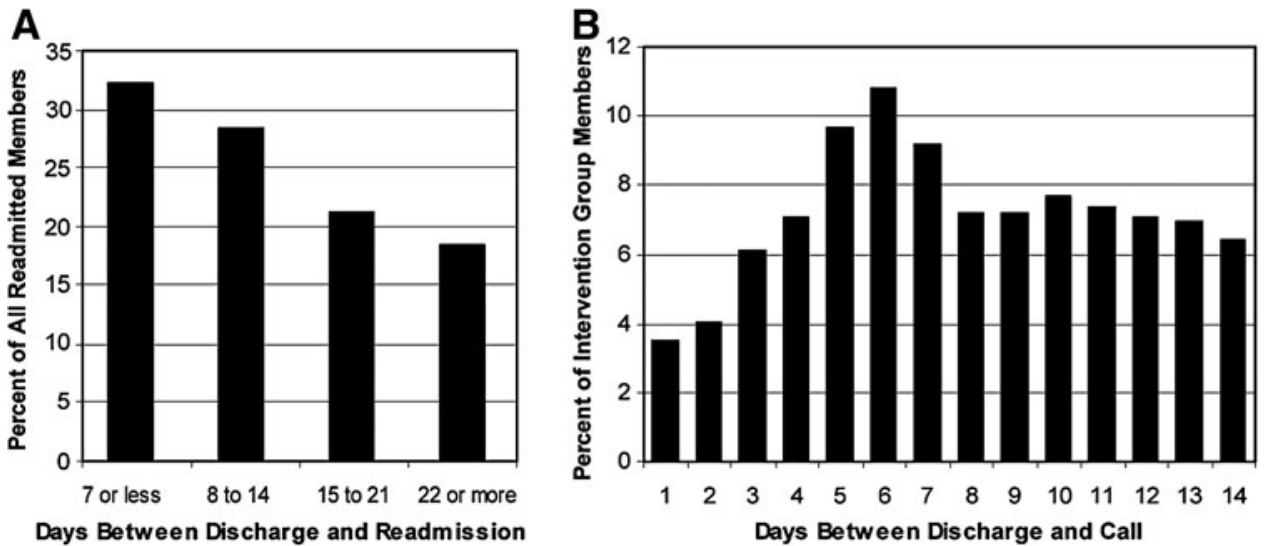

FIG. 3. Distribution of readmission and discharge calls with respect to day of discharge. (A) the distribution of readmissions by time frame after discharge among the total population; (B) the distribution of calls by day after discharge for intervention group members. 


\begin{tabular}{|c|c|c|c|c|}
\hline & $\begin{array}{c}\text { OR } \\
\text { Point } \\
\text { Estimate }\end{array}$ & $\begin{array}{l}95 \% \text { Wald } \\
\text { Confidence } \\
\text { Limits }\end{array}$ & $\begin{array}{c}\mathrm{P} \\
\text { value }\end{array}$ & \\
\hline Age at hospitalization & 1.193 & 1.144 & 1.244 & $<0.001$ \\
\hline Sex & 1.279 & 1.180 & 1.385 & $<0.001$ \\
\hline $\begin{array}{l}\text { Initial hospitalization } \\
\text { length of stay }\end{array}$ & 1.246 & 1.212 & 1.280 & $<0.001$ \\
\hline Readmission window & 1.048 & 1.020 & 1.077 & $<0.001$ \\
\hline Not called & 1.259 & 1.007 & 1.573 & $0.043^{*}$ \\
\hline
\end{tabular}

${ }^{*} P$ value determined from multiple logistic regression modeling with adjustment for age, sex, initial hospitalization length of stay, and readmission window.

subsequent hospital admissions and/or readmissions that occurred more than 30 days after the initial hospital discharge were not included in this study, although the program may have created a positive impact on these admissions as well.

A primary challenge for any telephonic program is contacting the entire target population. In the case of this program, connectivity is impacted by the location to which a member is discharged. For example, if patients enter a rehabilitation facility or stay with a family member after leaving the hospital, it is not possible to reach them using their home phone number. Furthermore, wrong phone numbers and delayed notification of hospital discharges from the health plan impedes the successful and timely delivery of calls to all discharged members. Because nearly a third of readmissions occur within a week of discharge, the ability to reach a discharged patient quickly is paramount to the overall success of the telephonic intervention. Despite these challenges, the telephonic model employed here produced significant reductions in readmissions. However, there is still opportunity to enhance the impact of programs such as this one with more thorough and efficient data exchange.

Table 3. Estimated Cost Savings as a Result of the Hospital Discharge Call Campaign

Number of called members 420 who were readmitted

Multiplied by the odds of readmission if not called

Estimated number of readmissions if not called

Minus the actual number of readmissions in intervention group

Estimated increase in readmissions if not called

Multiplied by the estimated cost per preventable readmission in $2008^{*}$

Estimated savings in study population as a result of discharge call campaign

*The cost per preventable readmission was based on work by Friedman and Basu and was adjusted to reflect nationwide trends and for inflation. ${ }^{11,24}$
There are a few limitations to this study that should be taken into consideration. First, it was not possible to determine conclusively that the impact on readmissions was solely the result of this call campaign without contribution by other efforts at reducing readmissions that may have differed between the intervention and comparison groups. For example, these members may have received phone calls or other outreach outside the scope of this study as a routine part of the disease management programs in which they were enrolled. Also, prospective studies are generally considered to be optimal because they often provide a greater balance between groups being compared with respect to variables known and unknown, to be related to the outcome of interest. We were only able to adjust for factors known to be associated with the likelihood of readmission, however, due to the retrospective nature of this study. Finally, because there was no direct method of controlling for the severity of disease, LOS was used as a proxy in the statistical model because this variable correlates with clinical risk. ${ }^{14}$

Although there are numerous studies of primary care initiatives that have demonstrated a reduction in readmissions, these have relied on the extended capacity of hospitals and clinics to reach out to patients following discharge. ${ }^{20-23}$ The results presented here demonstrate that telephonic counseling delivered shortly after hospital discharge can successfully reduce readmissions as well. Because telephonic-based approaches can be efficiently scaled and delivered, they offer a worthy option to mitigate the morbidity and cost associated with hospital readmissions. The importance of timing as a consideration in this intervention reveals an opportunity to improve the impact of programs such as this one, as well as a direction for future research. In conclusion, this study reveals the potential impact that focused telephonic follow-up subsequent to an initial hospital discharge can have on reducing preventable hospital readmissions, thereby minimizing the considerable costs associated with those readmissions.

\section{Author Disclosure Statement}

All authors of this paper are employees of Healthways, Inc., a provider of population health management services, including the intervention described in this paper.

\section{References}

1. Weinberger M, Oddone EZ, Henderson WG. Does increased access to primary care reduce hospital readmissions? Veterans Affairs Cooperative Study Group on Primary Care and Hospital Readmission. N Engl J Med 1996;334:14411447.

2. Douglas SL, Daly BJ, Kelley CG, O’Toole E, Montenegro H. Chronically critically ill patients: Health-related quality of life and resource use after a disease management intervention. Am J Crit Care 2007;16:447-457.

3. Benbassat J, Taragin M. Hospital readmissions as a measure of quality of health care: Advantages and limitations. Arch Intern Med 2000;160:1074-1081.

4. Bell CM, Schnipper JL, Auerbach AD, et al. Association of communication between hospital-based physicians and primary care providers with patient outcomes. J Gen Intern Med 2009;24:381-386. 
5. Jencks SF, Williams MV, Coleman EA. Rehospitalizations among patients in the Medicare fee-for-service program. N Engl J Med 2009;360:1418-1428.

6. Epstein AM. Revisiting readmissions-Changing the incentives for shared accountability. N Engl J Med 2009;360: 1457-1459.

7. Weissman JS, Ayanian JZ, Chasan-Taber S, Sherwood MJ, Roth C, Epstein AM. Hospital readmissions and quality of care. Med Care 1999;37:490-501.

8. Mulvany C. Preventable readmissions: A prime target for reform. Healthc Financ Manage 2009;63:32-34.

9. Iglehart JK. Reform and the health care workforce-Current capacity, future demand. 2009;361:e38.

10. Evans M. System overload. Rising demand at clinics straining resources. Mod Healthc 2009;39:33-34.

11. Friedman B, Basu J. The rate and cost of hospital readmissions for preventable conditions. Med Care Res Rev 2004;61:225-240.

12. Medicare Payment Advisory Commission. Promoting Greater Efficiency in Medicare. Washington, DC: Medicare Payment Advisory Commission (MedPAC); June 2007.

13. Anderson MA, Helms LB, Hanson KS, DeVilder NW. Unplanned hospital readmissions: A home care perspective. Nurs Res 1999;48:299-307.

14. MaWhinney S, Brown ER, Malcolm J, et al. Identification of risk factors for increased cost, charges, and length of stay for cardiac patients. Ann Thorac Surg 2000;70:702-710.

15. DeVol R, Bedroussian A. An Unhealthy America: The Economic Burden of Chronic Disease. Santa Monica, CA: Milken Institute; 2007.

16. Bauer M, Fitzgerald L, Haesler E, Manfrin M. Hospital discharge planning for frail older people and their family. Are we delivering best practice? A review of the evidence. J Clin Nurs 2009;18:2539-2546.

17. Centers for Disease Control and Prevention. The Burden of Chronic Diseases and their Risk Factors: National and State Perspectives. Atlanta, GA: U.S. Department of Health and Human Services, Centers for Disease Control and Prevention; 2004.
18. Chrusch CA, Olafson KP, McMillan PM, Roberts DE, Gray PR. High occupancy increases the risk of early death or readmission after transfer from intensive care. Crit Care Med 2009;37:2753-2758.

19. Terra SM. What can claims data tell the case manager? Prof Case Manag 2008;13:195-208.

20. Daly BJ, Douglas SL, Kelley CG, O'Toole E, Montenegro H. Trial of a disease management program to reduce hospital readmissions of the chronically critically ill. Chest 2005; 128:507-517.

21. Phillips CO, Wright SM, Kern DE, Singa RM, Shepperd S, Rubin HR. Comprehensive discharge planning with postdischarge support for older patients with congestive heart failure: A meta-analysis. JAMA 2004;291:1358-1367.

22. Phillips CO, Singa RM, Rubin HR, Jaarsma T. Complexity of program and clinical outcomes of heart failure disease management incorporating specialist nurse-led heart failure clinics. A meta-regression analysis. Eur J Heart Fail 2005; 7:333-341.

23. Balaban RB, Weissman JS, Samuel PA, Woolhandler S. Redefining and redesigning hospital discharge to enhance patient care: a randomized controlled study. J Gen Intern Med 2008;23:1228-1233.

24. The Henry J. Kaiser Family Foundation. Massachusetts: Average annual percent growth in health care expenditures by state of residence, 1991-2004. Available at: http:// statehealthfacts.org / profileind.jsp?ind $=595 \& \mathrm{cat}=5 \& \mathrm{rgn}=23$. Accessed October 16, 2009.

Address correspondence to; Elizabeth Y. Rula, Ph.D. Center for Health Research Healthways, Inc 701 Cool Springs Boulevard Franklin, TN 37067

E-mail: elizabeth.rula@healthways.com 